\title{
Sobrevida de pacientes com doença falciforme diagnosticados durante a triagem neonatal: revisão sistemática
}

\author{
Survival of sickle cell disease patients diagnosed during newborn screening: systematic review \\ Supervivencia de los pacientes con anemia de células falciformes diagnosticados durante el cribado
}

neonatal: revisión sistemática

Recebido: 11/08/2021 | Revisado: 18/08/2021 | Aceito: 23/08/2021 | Publicado: 24/08/2021

Carolina Mariano Pompeo

ORCID: https://orcid.org/0000-0003-4454-0140

Universidade Federal de Mato Grosso do Sul, Brasil

E-mail: carolmpompeo@gmail.com

Marcos Antonio Ferreira Júnior

ORCID: https://orcid.org/0000-0002-9123-232X

Universidade Federal de Mato Grosso do Sul, Brasil

E-mail: marcos_nurse@hotmail.com

Andreia Insabralde de Queiroz Cardoso

ORCID: https://orcid.org/0000-0002-9431-7484

Universidade Federal de Mato Grosso do Sul, Brasil

E-mail: andreiaqc@gmail.com

Mercy da Costa Souza

ORCID: https://orcid.org/0000-0001-9323-599X

Universidade Federal de Mato Grosso do Sul, Brasil

E-mail: me.enfmercy@gmail.com

Felipe Machado Mota

ORCID: https://orcid.org/0000-0001-6094-3404 Universidade Federal de Mato Grosso do Sul, Brasil

E-mail: felipemachadomota@gmail.com

Maria Lúcia Ivo

ORCID: https://orcid.org/0000-0003-2993-4758

Universidade Federal de Mato Grosso do Sul, Brasil

E-mail:marialuciaivoms@gmail.com

\begin{abstract}
Resumo
Objetivo: Analisar os dados relativos à sobrevida de pacientes com doença falciforme diagnosticados durante o processo de triagem neonatal. Método: Revisão sistemática, realizada nas bases eletrônicas Current Index to Nursing and Allied Health Literature, Cochrane Library, National Library of Medicine, Scopus e Web of Science com descritores controlados indexados no Medical Subject Headings. Sem delimitação temporal ou de idioma. Resultados: Sete artigos compuseram a amostra final, todos publicados em inglês e de delineamento de coorte. A avaliação de risco de vieses pelo Newcastle Otawa Scale indicou que seis artigos foram considerados de alta qualidade. Foram estudadas as taxas de sobrevida global, estratificadas por genótipos, eventos clínicos agudos, sexo e níveis de hemoglobina, além da sobrevida livre de eventos. A sobrevida global apresentou variação entre $100 \%$ aos seis meses e $85,6 \%$ aos 18 anos, enquanto para o genótipo $\mathrm{S} \beta^{\circ}$ foi estimada em $55,2 \%$ aos 40 anos. Conclusão: Houve diminuição da sobrevida após os 30 anos de idade, entretanto, com aumento anual entre os períodos estudados. A elaboração de políticas públicas com inserção da comunidade no contexto de suas ações pode auxiliar na manutenção das estratégias de melhoria da sobrevida desta população.
\end{abstract}

Palavras-chave: Doença falciforme; Triagem neonatal; Análise de sobrevida; Epidemiologia; Enfermagem baseada em evidências.

\begin{abstract}
Objective: To analyze the survival data of patients with sickle cell disease diagnosed during the neonatal screening process. Method: Systematic review, carried out in the electronic databases Current Index to Nursing and Allied Health Literature, Cochrane Library, National Library of Medicine, Scopus, and Web of Science with controlled descriptors indexed in the Medical Subject Headings. No time or language constraints. Results: Seven articles composed the final sample, all published in English and with a cohort design. The assessment of the risk of bias by the Newcastle Ottawa Scale indicated that six articles were considered of high quality. Overall survival rates, stratified by genotypes, acute clinical events, sex, and hemoglobin levels, in addition to event-free survival were studied. Overall
\end{abstract}


survival varied between $100 \%$ at six months and $85.6 \%$ at 18 years, while the $\mathrm{S} \beta^{\circ}$ genotype was estimated at $55.2 \%$ at 40 years. Conclusion: However, there was a decrease in survival after 30 years of age, with an annual increase between the periods studied. The development of public policies with the inclusion of the community in the context of its actions can help maintain strategies to improve the survival of this population.

Keywords: Sickle cell disease; Neonatal screening; Survival Analysis; Epidemiology; Evidence-based nursing.

\section{Resumen}

Objetivo: Analizar los datos de supervivencia de los pacientes con anemia de células falciformes diagnosticados durante el proceso de cribado neonatal. Método: Revisión sistemática, realizada en las bases de datos electrónicas Current Index to Nursing and Allied Health Literature, Cochrane Library, National Library of Medicine, Scopus y Web of Science con descriptores controlados indexados en Medical Subject Headings. Sin limitaciones de tiempo ni de idioma. Resultados: Siete artículos compusieron la muestra final, todos publicados en inglés y con un diseño de cohorte. La evaluación del riesgo de sesgo mediante la escala de Newcastle Ottawa indicó que seis artículos se consideraron de alta calidad. Se estudiaron las tasas de supervivencia global, estratificadas por genotipos, eventos clínicos agudos, sexo y niveles de hemoglobina, además de la supervivencia libre de eventos. La supervivencia global varió entre el $100 \%$ a los seis meses y el $85,6 \%$ a los 18 años, mientras que para el genotipo $\mathrm{S} \beta^{\circ}$ se estimó en un 55,2\% a los 40 años. Conclusión: Sin embargo, hubo una disminución de la supervivencia a partir de los 30 años, con un aumento anual entre los períodos estudiados. El desarrollo de políticas públicas con la inclusión de la comunidad en el contexto de sus acciones puede ayudar a mantener estrategias para mejorar la supervivencia de esta población.

Palabras clave: Enfermedad de célula falciforme; Cribado Neonatal; Análisis de supervivencia; Epidemiología; Enfermería basada en la evidencia.

\section{Introdução}

A doença falciforme (DF) consiste em um distúrbio genético de caráter hereditário que envolve diversas condições hematológicas que decorrem da mutação genética da cadeia beta globina e que podem resultar na formação de eritrócitos anormais. Na presença de desoxigenação esses eritrócitos podem sofrer alterações em sua capacidade de deformabilidade e de carrear gases sanguíneos e culminar em hemólise e quadros de vaso oclusão (Frédéric B Piel, Steinberg, \& Rees, 2017; Sundd, Gladwin, \& Novelli, 2019). Entre as condições hematológicas associadas à DF, a forma mais comum e mais grave da doença é a anemia falciforme (AF) (Ware, de Montalembert, Tshilolo, \& Abboud, 2017).

Dados do estudo Global Burden of Disease atualizados pelo site Institute for Health Metrics and Evaluation (IHME) estimaram a taxa de mortalidade esperada para todos os países do mundo (Piel, Hay, Gupta, Weatherall, \& Williams, 2013; Institute for Health Metrics and Evaluation, 2018). A taxa de mortalidade por DF observada nos Estados Unidos da América é de 0,23 por 100.000 habitantes, enquanto a taxa de mortalidade esperada seria de 0,0043. Para Burkina Faso, país com economia e expectativa de vida inversa ao primeiro, a taxa de mortalidade observada é de 14,27 enquanto a esperada seria de 0,27 por 100.000 habitantes (Institute for Health Metrics and Evaluation, 2018). É possível observar que mesmo entre os países mais ricos a taxa de mortalidade por DF observada foi muito superior à esperada o que torna imperativa a implementação de medidas de diagnóstico e tratamento precoce a essa população.

Há indicações de que a sobrevida de pacientes com DF em países de alta renda obteve grandes melhorias nos últimos anos em decorrência de diversos fatores relativos ao acesso aos serviços de saúde (Gardner et al., 2016). Mesmo assim, a DF é considerada um problema global, a qual requer que os gestores concentrem esforços para diminuir as mortes evitáveis. As ações devem ocorrer prioritariamente em países de baixa e média renda, com vistas ao planejamento adequado de políticas nacionais específicas aos indivíduos com DF (Frederic B Piel, Hay, Gupta, Weatherall, \& Williams, 2013; McGann, 2016).

Os programas de triagem neonatal se apresentam como proposta de diagnóstico e intervenção precoces, ainda nos primeiros meses de vida. Um estudo realizado na Bélgica observou diminuição nas infecções com melhoria na sobrevida, que pode ser atribuída a antibioticoterapia iniciada precocemente (Lê et al., 2018). 
Outro estudo de revisão sistemática também encontrou evidência de aumento na sobrevida de pacientes com DF diagnosticados pela triagem neonatal em decorrência das medidas precoces implementadas logo após o diagnóstico, entretanto um único estudo foi analisado (Runkel et al., 2020) o que torna necessária a realização de novas pesquisas acerca da temática.

No Brasil, além do Programa Nacional de Triagem Neonatal (PNTN) implantado em todo território nacional no ano de 2001 (Brasil, 2002), outros fatores podem ter contribuído para o aumento da sobrevida desta população, como o acesso a medicamentos e imunobiológicos especiais, o acompanhamento por equipe multidisciplinar, a garantia do seguimento das pessoas diagnosticadas com hemoglobinopatias pelo PNTN, entre outros, garantidos pelas diretrizes para a Política Nacional de Atenção Integral às Pessoas com Doença Falciforme e outras Hemoglobinopatias no ano de 2005 (Brasil, 2005).

Diante do exposto, este estudo se propõe a investigar os artigos publicados sobre a temática, a fim de produzir indicadores que possam colaborar com a análise de sobrevida desta população, por meio da seguinte questão norteadora: Qual a sobrevida de pacientes com DF diagnosticados durante o processo de triagem neonatal?

\section{Metodologia}

Trata de um estudo de revisão sistemática da literatura, realizada e relatada de acordo com os itens das diretrizes Preferred Reporting Items for Systematic Reviews and Meta-Analyses (PRISMA) (Moher et al., 2015) e Meta-analysis Of Observational Studies in Epidemiology (MOOSE) (Stroup et al., 2000), além da utilização de um instrumento estruturado de registro de dados e acompanhamento.

\section{Critérios de elegibilidade}

Para elaboração da questão norteadora do estudo foi utilizado o acrônimo PICOS (Kloda \& Bartlett, 2013), sendo "P" (Population) se refere aos pacientes com DF; "I" (Intervention) a triagem neonatal; "C" (Comparison) a sobrevida das coortes por período de acompanhamento; "O" (Outcome) aos desfechos relacionados à sobrevida em DF e "S" (Study desing) aos estudos observacionais.

\section{Fonte de informação}

A busca nas bases de dados eletrônicas foi realizada em 30 de junho de 2020, por dois pesquisadores independentes com uso do Proxy da Universidade Federal de Mato Grosso do Sul (UFMS) por meio do Portal de Periódicos da Coordenação de Aperfeiçoamento de Pessoal de Nível Superior (CAPES). Após testagem dos cruzamentos em todas as bases que veiculam publicações acerca da temática foram adotadas apenas as que recuperaram estudos que respondiam à questão norteadora, que seguem: Current Index to Nursing and Allied Health Literature (CINAHL), Cochrane Library, National Library of Medicine (Medline via PubMed), Elservier's Scopus (SCOPUS) e Web of Science.

\section{Estratégias de busca}

Foram utilizados descritores controlados indexados no Medical Subject Headings (MeSH) com aplicação do operador booleano "AND" entre os termos. Não foi utilizado o conector "OR" em decorrência da não utilização de entry terms para a estruturação das estratégias. Os detalhes das buscas estão descritos no Quadro 1. 
Quadro 1. Estratégias de busca para as bases de dados adotadas e total de materiais inicialmente recuperados, Campo Grande, Mato Grosso do Sul, Brasil, 2021.

\begin{tabular}{|llc|}
\hline Base de dados & Estratégia de Busca & Resultados \\
\hline CINAHL & 1\# “Anemia, Sickle Cell” AND “Survival Rate” & 118 \\
\hline PubMed & 1\# ("Anemia, Sickle Cell"[Mesh]) AND "Survival Rate"[Mesh] & 161 \\
\hline Scopus & 1\# ( "Anemia, Sickle Cell" ) AND ( "Survival Rate" ) AND ( LIMIT- & 135 \\
\hline Web of Science & TO ( DOCTYPE, "ar" ) ) & 03 \\
\hline Cochrane Library & 1\# AS=(Anemia, Sickle Cell AND Survival Rate) \\
\hline
\end{tabular}

Fonte: Autores.

\section{Seleção dos estudos e processo de coleta de dados}

Foram incluídos artigos completos de estudos observacionais que continham análise de sobrevida de indivíduos com DF diagnosticados durante o processo de triagem neonatal. Não foi estabelecido limite de idioma ou recorte de tempo. Foram excluídos estudos que realizaram análise de pacientes com diagnóstico tardio, além de análises específicas para transplante medula óssea e tratamentos cirúrgicos, além das pesquisas com modelos animais.

A seleção nas bases foi realizada no dia 30 de junho de 2020, com exclusão automática de duplicatas mediante uso do software Mendeley, seguida pela leitura de títulos e resumos pelo Rayyan QCRI (Ouzzani, Hammady, Fedorowicz, \& Elmagarmid, 2016) pelos pesquisadores, de forma independente.

Após a triagem foi realizada a leitura dos textos completos dos estudos selecionados para verificação dos critérios de inclusão/exclusão de 01 a 03 de julho de 2020. Após essa etapa, no período de 04 a 08 de julho de 2020 procedeu-se a leitura na íntegra dos selecionados com extração dos dados para o software Microsoft Excel.

Todo processo de triagem dos artigos, avaliação da qualidade metodológica e risco de vieses foi realizado por dois pesquisadores de forma independente. As discordâncias foram resolvidas por consenso após a comparação dos resultados e verificação das diferenças dos achados.

\section{Extração e análise dos dados}

Para extração e análise dos dados foi elaborado um instrumento específico para esse estudo composto pelas seguintes variáveis: delineamento metodológico, país, idioma, área de conhecimento, instituição sede, revista ou jornal científico de publicação, objetivo, população, comparador/controle, amostra, sexo, faixa etária, genótipo, período de acompanhamento, perda de seguimento, desfecho (óbito/sobrevida), tipo de estudo, classificação do nível de evidência, limitações do estudo e conflitos de interesses, além das taxas de sobrevida encontradas, objeto desse estudo.

\section{Risco de viés em cada estudo}

Para a análise da qualidade metodológica e avaliação do risco de vieses em cada estudo primário foi realizada com utilização da escala Newcastle Otawa Scale (NOS), um sistema estrelar (0 a 9) com avaliação de três domínios: seleção, comparabilidade e resultados (Wells et al., 2014). 


\section{Medidas de sumarização}

Os desfechos para análise de subgrupo foram realizados com base no genótipo dos pacientes descrito nos estudos, assim como a taxa de letalidade das coortes. As medianas de sobrevida global, por genótipos $\mathrm{SS} / \mathrm{S} \beta^{\circ}$ e $\mathrm{SC} / \mathrm{S} \beta+$ foram calculadas, além da mediana de sobrevida estratificada por período de acompanhamento das coortes acompanhadas antes e após o ano de 1981. O corte temporal para estratificação e cálculo da mediana de sobrevida foi escolhido por englobar de forma igualitária o maior número de coortes de follow up antes e após o período escolhido para a análise dos estudos encontrados.

\section{Síntese dos resultados}

A síntese dos dados foi realizada de maneira narrativa com base nos resultados relativos aos desfechos de interesse desta revisão sistemática. Não foi realizada metanálise devido à heterogeneidade dos estudos incluídos, por este motivo a análise ocorreu por estatística descritiva dos achados quantitativos.

\section{Resultados}

\section{Seleção de estudos}

Foram recuperados inicialmente 425 artigos, com a retirada de 126 duplicados que foram considerados apenas uma vez e rastreados 299 por título e resumo. Destes, apenas 11 apresentaram critérios de elegibilidade, dos quais sete (A. Lee, Thomas, Cupidore, Serjeant, \& Serjeant, 1995; Quinn, Rogers, \& Buchanan, 2004; King et al., 2007; Telfer et al., 2007; Fernandes, Nélio Januário, Blanco Cangussu, Lino de Macedo, \& Borato Viana, 2010; Quinn, Rogers, McCavit, \& Buchanan, 2010; Serjeant et al., 2018) fizeram parte da amostra final dessa revisão sistemática. O resultado da busca encontra-se descrito na Figura 1, baseado no Flow Diagrama PRISMA. 
Figura 1. Fluxograma de triagem dos estudos recuperados, Campo Grande, Mato Grosso do Sul, Brasil, 2021.

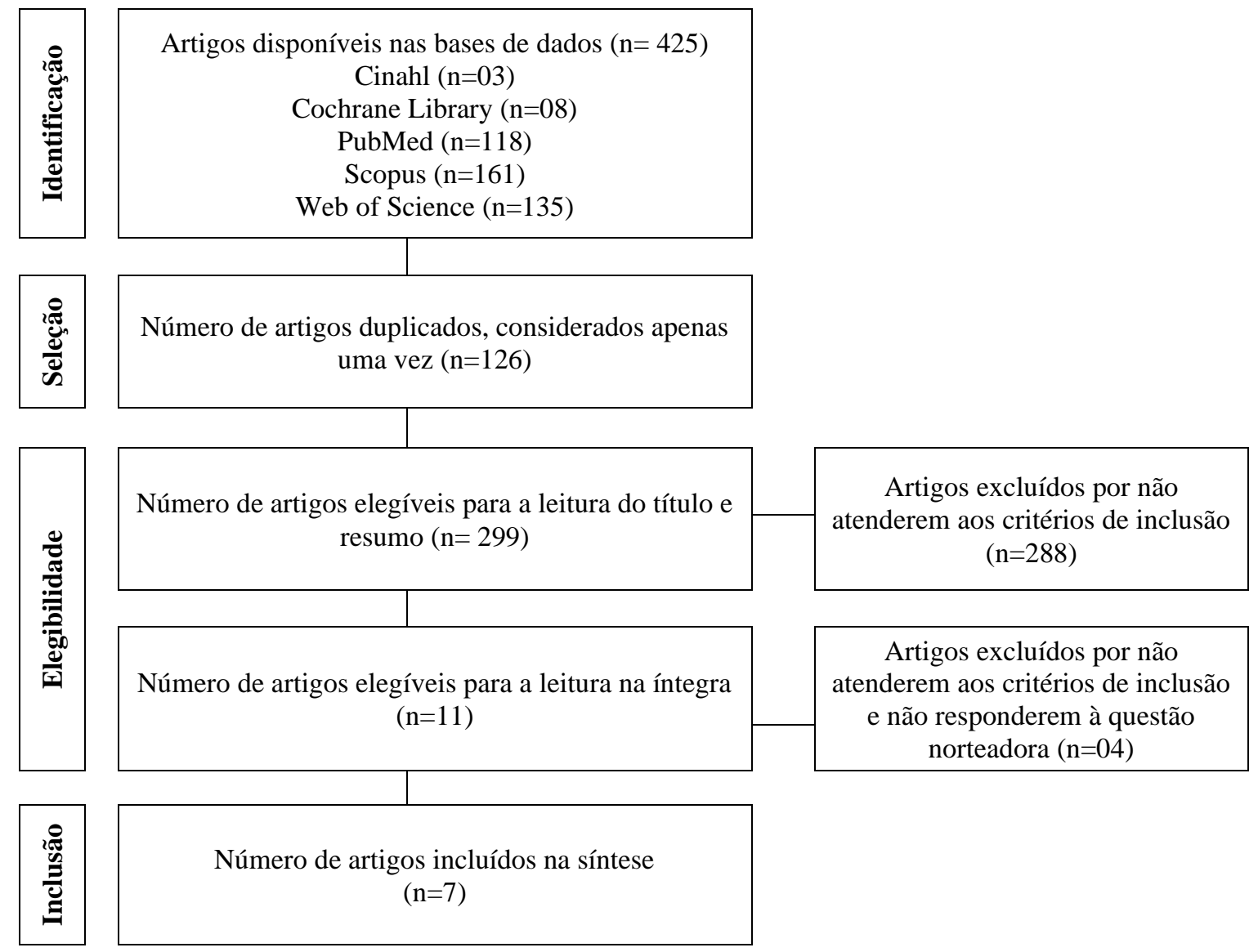

Fonte: Dados da pesquisa (2021).

\section{Características e resultados dos estudos}

Dos sete artigos que compuseram esta revisão sistemática, três tratavam de estudos realizados na Jamaica (A. Lee et al., 1995; King et al., 2007; Serjeant et al., 2018), dois nos Estados Unidos da América (Quinn et al., 2004; Quinn et al., 2010), um no Reino Unido (Telfer et al., 2007) e um no Brasil (Fernandes et al., 2010), todos publicados no idioma inglês. A maioria dos estudos (A. Lee et al., 1995; Quinn et al., 2004; Telfer et al., 2007; Quinn et al., 2010; Serjeant et al., 2018) foi conduzida com delineamento de coorte prospectiva $(71,73 \%)$ e apenas dois foram de coorte retrospectiva (28,27\%) (King et al., 2007; Fernandes et al., 2010).

A população dos estudos foi composta por crianças com diagnóstico de DF realizado durante a triagem neonatal. A sobrevida foi avaliada de forma isolada em três estudos (A. Lee et al., 1995; Fernandes et al., 2010; Serjeant et al., 2018), em outros quatro foi avaliada juntamente com outras variáveis como a morbimortalidade e a presença de eventos como o acidente vascular cerebral (AVC) e a sepse pneumocócica. Todos os estudos referiram melhorias na sobrevida e também na sobrevida livre de eventos, conforme apresentado no Quadro 2. 
Quadro 2. Caracterização dos estudos relacionados a sobrevida dos pacientes com doença falciforme diagnosticados durante a triagem neonatal, Campo Grande, Mato Grosso do Sul, Brasil, 2021.

\begin{tabular}{|c|c|c|c|c|}
\hline Objetivo & $\begin{array}{c}\text { População } \\
\text { (n) }\end{array}$ & $\begin{array}{c}\text { Comparador/ } \\
\text { Controle }\end{array}$ & Desfecho & $\begin{array}{c}\text { Delineamento } \\
\text { do estudo }\end{array}$ \\
\hline $\begin{array}{l}\text { Analisar se intervenções simples } \\
\text { em uma clínica melhoram a } \\
\text { sobrevida na DF (A. Lee et al., } \\
\text { 1995). }\end{array}$ & $\begin{array}{c}\text { Crianças } \\
(315)\end{array}$ & $\begin{array}{l}\text { Sobrevida das coortes } \\
\text { nos períodos } 1973 \\
1975 ; 1975 \text { a } 1979 \text { e } \\
1979 \text { a } 1981\end{array}$ & $\begin{array}{l}\text { Melhora da sobrevida global ao } \\
\text { comparar o primeiro, o segundo } \\
\text { e o último terço do estudo e da } \\
\text { sobrevida por sequestro } \\
\text { esplênico agudo e sepse por } \\
\text { meningite pneumocócica. }\end{array}$ & $\begin{array}{c}\text { Coorte } \\
\text { prospectiva }\end{array}$ \\
\hline $\begin{array}{l}\text { Avaliar o programa de } \\
\text { rastreamento } \\
\text { hemoglobinopatia para DF em } \\
\text { recém-nascidos na Jamaica (King } \\
\text { et al., 2007). }\end{array}$ & $\begin{array}{c}\text { Crianças } \\
(395)\end{array}$ & $\begin{array}{l}\text { Sobrevida das coortes } \\
\text { nos períodos } 1973 \\
1975 ; 1975 \text { a } 1979 ; \\
1979 \text { a } 1981 \text { e } 1995 \text { a } \\
2006\end{array}$ & $\begin{array}{l}\text { Melhora da sobrevida em } \\
\text { comparação com o estudo de } \\
\text { coorte jamaicano com menor } \\
\text { proporção de internações e } \\
\text { episódios de doença grave no } \\
\text { grupo de estudo. }\end{array}$ & $\begin{array}{c}\text { Coorte } \\
\text { retrospectiva }\end{array}$ \\
\hline $\begin{array}{l}\text { Analisar os nascimentos com DF } \\
\text { para fornecer valores de } \\
\text { morbimortalidade no contexto do } \\
\text { Reino Unido e a diferença com os } \\
\text { Estados Unidos e Jamaica (Telfer } \\
\text { et al., 2007). }\end{array}$ & $\begin{array}{c}\text { Crianças } \\
(252)\end{array}$ & $\begin{array}{l}\text { Sobrevida; } \\
\text { Sobrevida das coortes } \\
\text { nos períodos } 1983 \text { a } \\
2006 ; 1978-1988 ; \\
1979-1981 ; 1983- \\
2002 ; 1983-2006\end{array}$ & $\begin{array}{l}\text { Houve aumento da sobrevida } \\
\text { quando comparado a outras } \\
\text { coortes relatadas. }\end{array}$ & $\begin{array}{c}\text { Coorte } \\
\text { prospectiva }\end{array}$ \\
\hline $\begin{array}{l}\text { Determinar dados de } \\
\text { sobrevivência contemporâneos } \\
\text { para crianças com DF que melhor } \\
\text { refletem o impacto da terapia } \\
\text { moderna (Quinn et al., 2004). }\end{array}$ & $\begin{array}{c}\text { Crianças } \\
\text { (711) }\end{array}$ & $\begin{array}{l}\text { Sobrevida das coortes } \\
\text { nos períodos } 1983- \\
2002\end{array}$ & $\begin{array}{l}\text { Melhor sobrevida entre o grupo } \\
\text { de hemoglobinopatias } \mathrm{SC} / \mathrm{S} \beta+ \\
\text { comparado ao grupo } \mathrm{SS} / \mathrm{S} \beta^{\circ} \text {. }\end{array}$ & $\begin{array}{c}\text { Coorte } \\
\text { prospectiva }\end{array}$ \\
\hline $\begin{array}{l}\text { Estimar a sobrevida na DF } \\
\text { homozigótica por seleção } \\
\text { sintomática e verificar as causas } \\
\text { de morte em uma população pré- } \\
\text { hidroxiureia (Serjeant et al., } \\
\text { 2018). }\end{array}$ & $\begin{array}{c}\text { Crianças } \\
(311)\end{array}$ & $\begin{array}{l}\text { Sobrevida das coortes } \\
\text { nos períodos 1973- } \\
2016\end{array}$ & $\begin{array}{l}\text { A sobrevida aos } 40 \text { anos foi } \\
\text { menor naqueles com menores } \\
\text { níveis de hemoglobina, alta } \\
\text { contagem de células nucleadas } \\
\text { total e histórico de dactilite. }\end{array}$ & $\begin{array}{c}\text { Coorte } \\
\text { prospectiva }\end{array}$ \\
\hline $\begin{array}{l}\text { Estimar a } \quad r \text { sobrevida } \\
\text { contemporânea de } 18 \text { anos em } \\
\text { recém-nascidos com DF e } \\
\text { documentar nas causas e idades } \\
\text { da morte (Quinn et al., 2010). }\end{array}$ & $\begin{array}{c}\text { Crianças } \\
(940)\end{array}$ & $\begin{array}{l}\text { Sobrevida das coortes } \\
\text { nos períodos 1983- } \\
\text { 1990; 1991-2000; } \\
\text { 2000-2007 }\end{array}$ & $\begin{array}{l}\text { Houve melhoria na sobrevida e } \\
\text { maioria das crianças com DF, } \\
\text { sobrevivem aos anos da } \\
\text { infância. }\end{array}$ & $\begin{array}{c}\text { Coorte } \\
\text { prospectiva }\end{array}$ \\
\hline $\begin{array}{l}\text { Descrever as mortes de crianças } \\
\text { com DF em Minas Gerais, Brasil } \\
\text { (Fernandes et al., 2010). }\end{array}$ & $\begin{array}{c}\text { Crianças } \\
(1.396)\end{array}$ & $\begin{array}{l}\text { Sobrevida das coortes } \\
\text { nos períodos 1998- } \\
2005\end{array}$ & $\begin{array}{lr}\text { Melhor sobrevida } & \text { entre } \\
\text { hemoglobinopatias } & \mathrm{SC} / \mathrm{S} \beta+ \\
\text { comparado a SS. } & \end{array}$ & $\begin{array}{l}\text { Coorte } \\
\text { retrospectiva }\end{array}$ \\
\hline
\end{tabular}

Nota $:$ DF = Doença falciforme. Fonte: Autores.

Um total de 4.320 indivíduos fizeram parte das pesquisas, a maioria apresentou genótipo HbSS (51,02\%). Vale ressaltar que em um estudo (Fernandes et al., 2010) a doença homozigótica foi investigada com o genótipo HbS $\beta^{\circ}$ e ao serem somados os genótipos $\mathrm{SS} / \mathrm{S} \beta^{\circ}$ de todas as coortes essa porcentagem passa a ser de 69,59\%. Foram registrados 324 óbitos, destes $221(68,21 \%)$ ocorreram em pacientes com genótipo HbSS.

O seguimento dos pacientes nos estudos apresentou mediana de 18 anos, com menor período de acompanhamento de sete (Fernandes et al., 2010) e maior de 43 anos (Serjeant et al., 2018). A maior perda de seguimento ficou em 24,1\% (Serjeant 
et al., 2018) e a menor em 2,5\% (A. Lee et al., 1995), embora dois estudos não tenham relatado este dado (King et al., 2007; Fernandes et al., 2010). A taxa de letalidade global calculada foi de 7,5\% (Tabela 1). A Tabela 2 e Figura 2 apresentam a sobrevida global e específica por genótipos.

Tabela 1. Descrição das amostras e genótipos, tempo de seguimento, perdas, óbitos e taxa de letalidade calculada dos estudos analisados, Campo Grande, Mato Grosso do Sul, Brasil, 2021.

\begin{tabular}{|c|c|c|c|c|c|c|c|c|c|}
\hline \multirow{2}{*}{ Autor (Ano) } & \multicolumn{6}{|c|}{ Genótipo } & \multirow{2}{*}{$\begin{array}{c}{ }^{*} \text { Follow } \\
\text { Up }\end{array}$} & \multirow[b]{2}{*}{ Perdas $(\%)$} & \multirow{2}{*}{$\begin{array}{c}\text { Óbitos } \\
\left({ }^{\dagger} \text { Let. \%) }\right.\end{array}$} \\
\hline & SS & SC & $\mathbf{S} \boldsymbol{\beta}^{\circ}$ & $\mathbf{S \beta}+$ & SD & $\mathbf{S S} / \mathbf{S} \beta^{\circ}$ & & & \\
\hline A. Lee et al. (1995) & 315 & - & - & - & - & - & 15 & $2,5 \%$ & $61(19,37 \%)$ \\
\hline King et al. (2007) & 395 & - & - & - & - & - & 11 & ${ }^{*} \mathrm{NI}$ & $07(1,77 \%)$ \\
\hline Telfer et al. (2007) & 180 & 64 & - & - & - & - & 23 & $2,8 \%$ & $02(0,79 \%)$ \\
\hline Quinn et al. (2004) & 431 & 215 & 17 & 48 & & & 18 & $15,6 \%$ & $25(3,52 \%)$ \\
\hline Serjeant et al. (2018) & 311 & - & - & - & - & - & 43 & $24,1 \%$ & $119(38,26 \%)$ \\
\hline Quinn et al. (2010) & 572 & 284 & 21 & 63 & - & - & 19 & $7,3 \%$ & $32(3,40 \%)$ \\
\hline Fernandes et al. (2010) & - & 555 & - & 67 & 10 & 764 & 07 & ${ }^{*} \mathrm{NI}$ & $78(5,59 \%)$ \\
\hline Total & 2.204 & 764 & 38 & 178 & 10 & 764 & - & - & $324(7,5 \%)$ \\
\hline
\end{tabular}

Nota: ${ }^{*}$ Follow up = Período de acompanhamento em anos; ${ }^{\dagger}$ Let. $=$ Taxa de letalidade calculada para as coortes; ${ }^{\star} \mathrm{NI}=$ Não informado. Fonte: Autores. 
Tabela 2. Probabilidade de sobrevida por genótipos da doença falciforme por idade (anos), Campo Grande, Mato Grosso do Sul, Brasil, 2021.

\begin{tabular}{|c|c|c|c|c|c|c|c|c|c|c|c|c|c|c|c|c|c|}
\hline \multirow{2}{*}{ Sobrevida das hemoglobinopatias } & \multicolumn{17}{|c|}{ Probabilidade de sobrevida por idade em anos (\%) } \\
\hline & $\mathbf{0 , 5}$ & 1 & 2 & 3 & 4 & 5 & 6 & 8 & 10 & 12 & 14 & 15 & 16 & 18 & 20 & 30 & 40 \\
\hline Coorte SS (1973 a 1975) (A. Lee et al., 1995) & - & 90 & 86 & 83 & - & 81 & - & - & 77 & - & - & 73 & - & - & - & - & - \\
\hline Coorte SS (1975 a 1979) (A. Lee et al., 1995) & - & 97 & 95 & 93 & - & 91 & - & - & 85 & - & - & 83 & - & - & - & - & - \\
\hline Coorte SS (1979 a 1981) (A. Lee et al., 1995) & - & 95 & 94 & 93 & - & 91 & - & - & 87 & - & - & 84 & - & - & - & - & - \\
\hline SS (1995 a 2006) ${ }^{(\text {King et al., 2007) }}$ & - & 99 & 99 & 99 & - & 98 & - & - & 91 & - & - & - & - & - & - & - & - \\
\hline SS 10/20 anos global (Telfer et al., 2007) & - & - & - & - & - & - & - & - & 97,9 & - & - & - & - & - & 97,9 & - & - \\
\hline DF$^{1}(1983-2002)^{(T e l f e r ~ e t ~ a l ., ~ 2007) ~}$ & - & - & - & - & - & - & - & - & - & - & - & - & - & 86 & - & - & - \\
\hline $\mathrm{DF}^{1}(1983-2006)$ (Telfer et al., 2007) & - & - & - & - & - & - & - & - & - & - & - & - & - & - & 99 & - & - \\
\hline Global SS/S $\beta^{\text {o (Quinn et al., 2004) }}$ & 100 & 99,5 & 98,5 & - & 97,7 & - & 95 & 94,6 & 94,6 & 94,6 & 93 & - & 91,7 & 85,6 & - & - & - \\
\hline Global SC/S $\beta+{ }^{\text {Quinn et al., 2004) }}$ & - & - & - & - & - & - & - & - & - & - & - & - & - & 97,4 & - & - & - \\
\hline Global relacionada DF1 (Quinn et al., 2004) & 100 & 99,8 & 99,5 & - & 98,6 & - & 96,3 & 95,9 & 95,9 & 95,9 & 94,9 & - & 93,6 & 93,6 & - & - & - \\
\hline $\mathrm{SC} / \mathrm{S} \beta+$ relacionada a $\mathrm{DF}^{1}$ (Quinn et al., 2004) & - & - & - & - & - & - & - & - & - & - & - & - & - & 100 & - & - & - \\
\hline SS (Serjeant et al., 2018) & - & 93,9 & - & - & - & 87,1 & - & - & 82,5 & - & - & - & - & 76,9 & 74,9 & 65,5 & 55,2 \\
\hline $\mathrm{SS} / \mathrm{S} \beta^{\mathrm{o}}$ (Quinn et al., 2010) & - & - & - & - & - & - & - & - & - & - & - & - & - & 93,9 & - & - & - \\
\hline $\mathrm{SC} / \mathrm{S} \beta+$ (Quinn et al., 2010) & - & - & - & - & - & - & - & - & - & - & - & - & - & 98,4 & - & - & - \\
\hline $\mathrm{SS} / \mathrm{S} \beta^{\circ}(1983-1990)$ (Quinn et al., 2010) & - & - & - & - & - & 96,8 & - & - & - & - & - & - & - & - & - & - & - \\
\hline $\mathrm{SS} / \mathrm{S} \beta^{\circ}$ (1991-2000) (Quinn et al., 2010) & - & - & - & - & - & 97,5 & - & - & - & - & - & - & - & - & - & - & - \\
\hline $\mathrm{SS} / \mathrm{S} \beta^{\circ}(2000-2007)$ (Quinn et al., 2010) & - & - & - & - & - & 99,2 & - & - & - & - & - & - & - & - & - & - & - \\
\hline $\mathrm{SS} / \mathrm{S} \beta^{\circ}$ (Fernandes et al., 2010) & - & 96,6 & - & 91,6 & - & 89,4 & - & - & - & - & - & - & - & - & - & - & - \\
\hline SC (Fernandes et al., 2010) & - & 98,7 & - & 98 & - & 97,7 & - & - & - & - & - & - & - & - & - & - & - \\
\hline $\mathrm{S} \beta+$ (Fernandes et al., 2010) & - & 96,8 & - & 94,7 & - & 94,7 & - & - & - & - & - & - & - & - & - & - & - \\
\hline SD (Fernandes et al., 2010) & - & 100 & - & 100 & - & 100 & - & - & - & - & - & - & - & - & - & - & - \\
\hline & & & & & & & & & & & & & & & & u & ela 2) \\
\hline
\end{tabular}


Research, Society and Development, v. 10, n. 11, e95101119329, 2021

(CC BY 4.0) | ISSN 2525-3409 | DOI: http://dx.doi.org/10.33448/rsd-v10i11.19329

\begin{tabular}{|c|c|c|c|c|c|c|c|c|c|c|c|c|c|c|c|c|c|}
\hline \multirow{2}{*}{ Sobrevida das hemoglobinopatias } & \multicolumn{17}{|c|}{ Probabilidade de sobrevida por idade em anos (\%) } \\
\hline & $\mathbf{0 , 5}$ & 1 & 2 & 3 & 4 & 5 & 6 & 8 & 10 & 12 & 14 & 15 & 16 & 18 & 20 & 30 & 40 \\
\hline Global todos os genótipos (Fernandes et al., 2010) & - & 97,5 & - & 94,3 & - & 93,1 & - & - & - & - & - & - & - & - & - & - & - \\
\hline Mediana de sobrevida global* & 100 & 97,3 & 96,8 & 94,3 & 98,2 & 94,7 & 95,7 & 95,3 & 89 & 95,3 & 94,0 & 83,0 & 92,7 & 93,8 & 97,9 & 65,5 & 55,2 \\
\hline Mediana sobrevida $\mathrm{SS} / \mathrm{S} \beta^{\circ *}$ & 100 & 96,6 & 95,0 & 93,0 & 97,7 & 91,0 & 95,0 & 94,6 & 87,0 & 94,6 & 93,0 & 83,0 & 91,7 & 85,6 & 86,4 & 65,5 & 55,2 \\
\hline Mediana sobrevida $\mathrm{SC} / \mathrm{S} \beta+*$ & - & 97,6 & - & 96,4 & - & 96,2 & - & - & - & - & - & - & - & 98,4 & - & - & - \\
\hline
\end{tabular}

Nota $:$ DF $=$ Doença falciforme. $*$ Mediana de sobrevida calculada para os grupos. Fonte: Autores. 
Figura 2. Probabilidade de sobrevida global e estratificada por período antes e após 1981, Campo Grande, Mato Grosso do Sul, Brasil, 2021.

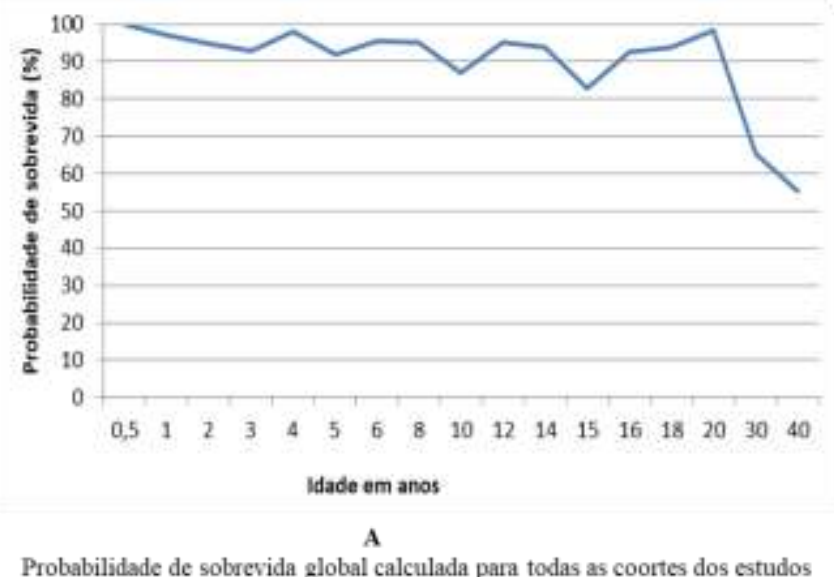

Probabilidade de sobrevida global calculada para todas as coortes dos estudos

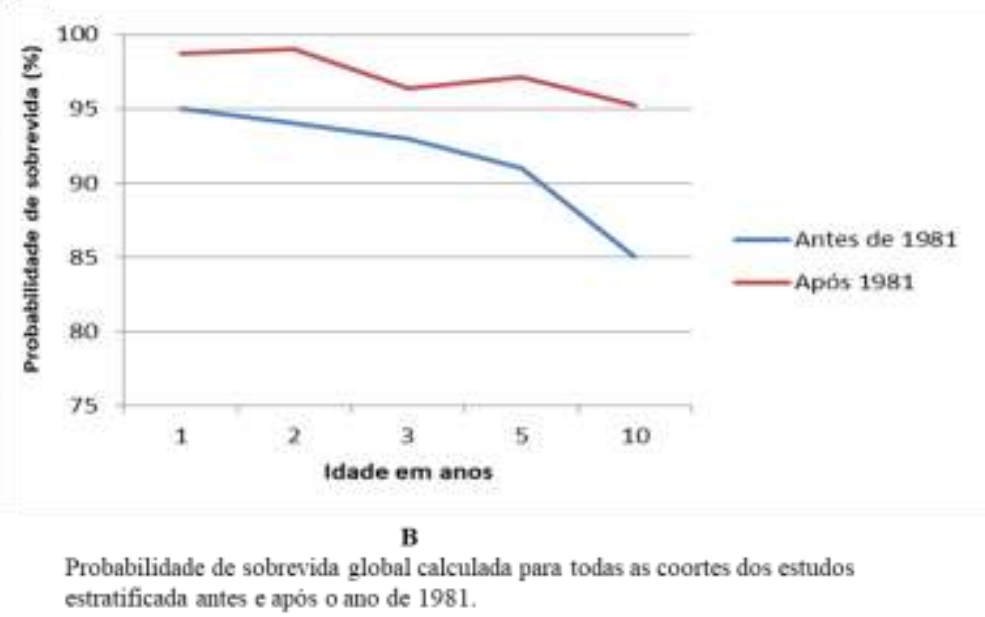

Fonte: Dados da pesquisa (2021).

A mediana de sobrevida global aos 18 anos foi de 93,8\%, para os genótipos $\mathrm{SS} / \mathrm{S} \beta^{\circ}$ e $\mathrm{SC} / \mathrm{S} \beta+$ foi de $85,6 \%$ e $98,4 \%$, respectivamente, enquanto para todas as coortes dos estudos aos seis meses de idade foi de $100 \%$ e declinou até próximo de $55 \%$ aos 40 anos. Ao ser estratificada por período de acompanhamento antes e após o ano de 1981, a mediana de sobrevida no primeiro período foi de $85 \%$ aos 10 anos e de cerca de $95 \%$ no segundo período para a mesma idade.

Além destes dados, outras informações não apresentadas na tabela são de igual importância. Em relação à sobrevida estratificada por sexo, esta foi discretamente maior para o masculino (77,5\%). Quando da estratificação por níveis de hemoglobina estes foram diretamente proporcionais à sobrevida, valores de $10 \mathrm{~g} / \mathrm{dL}$ no início da vida foram associados a uma sobrevida de $78 \%$, enquanto valores de $6 \mathrm{~g} / \mathrm{dL}$ representaram $56 \%$ aos 30 anos. Níveis elevados de células nucleadas totais (TNC) foram associados a uma sobrevida de $83 \%$ em qualquer idade.

A sobrevida estratificada por presença de eventos graves e não graves em relação aos períodos de 1973-1975, 19751979 e 1979-1981 apresentou melhora quando comparados os três grupos. A sobrevida com um ano de idade foi de 96,99 e 99\% para os três períodos respectivamente e aos 15 anos foi de 91, 97 e $98 \%$ na mesma ordem.

Já a sobrevida livre de eventos apresentou relação inversamente proporcional ao tempo de acompanhamento, com diminuição ao longo do período, a exemplo da sobrevida livre de AVC. Em pacientes SS/S $\beta^{\circ}$ a probabilidade de sobrevivência livre daquele evento era de $100 \%$ aos seis meses de vida e aos 18 anos chegou a 88,5, exceção aos genótipos SC/S $\beta+$ cuja probabilidade de sobrevivência permaneceu em 100\% aos 18 anos. Para os casos que apresentaram eventos de dactilite a sobrevida no primeiro ano de vida foi de $78,2 \%$, mas aos cinco anos declinou para $51,7 \%$.

\section{Risco de viés}

A avaliação do risco de viés pela escala NOS (Wells et al., 2014) resultou em três artigos com o total de nove estrelas (A. Lee et al., 1995; Quinn et al., 2004; Telfer et al., 2007), um estudo com oito (Quinn et al., 2010), dois com sete (King et al., 2007; Serjeant et al., 2018) e um com seis estrelas (Fernandes et al., 2010). Seis artigos (A. Lee et al., 1995; Quinn et al., 2004; Telfer et al., 2007; Fernandes et al., 2010; Quinn et al., 2010; Serjeant et al., 2018) pontuaram com quatro estrelas no item seleção, apenas um (King et al., 2007) apresentou três estrelas nesta avaliação. Em relação à comparabilidade, quatro artigos (A. Lee et al., 1995; Quinn et al., 2004; King et al., 2007; Telfer et al., 2007) pontuaram com duas estrelas e três (Fernandes et 
al., 2010; Quinn et al., 2010; Serjeant et al., 2018) com uma estrela cada. Para o último item de avaliação, o desfecho, quatro (A. Lee et al., 1995; Quinn et al., 2004; Telfer et al., 2007; Quinn et al., 2010) artigos pontuaram com três estrelas, dois (King et al., 2007; Serjeant et al., 2018) com duas e apenas um (Fernandes et al., 2010) pontuou com uma estrela.

\section{Discussão}

Esta revisão buscou avaliar a sobrevida de indivíduos com DF diagnosticados pelos programas de triagem neonatal. Atualmente, tais programas estão implantados em muitos países do mundo, a exemplo do Brasil, onde o Programa Nacional de Triagem Neonatal para diagnóstico das hemoglobinopatias ocorre desde 2001. Estudos brasileiros apontam uma cobertura estimada de 95\% quando avaliadas crianças até dois anos (Mallmann, Tomasi, \& Boing, 2020) e de 90\% entre os nascidos vivos (Kikuchi et al., 2018).

Mesmo assim, várias nações de baixa renda pelo mundo ainda enfrentam problemas na triagem neonatal, como alocação de recursos, treinamento de pessoal, materiais de consumo, ausência de mecanismos de coleta regulares e até mesmo fornecimento de energia elétrica (Nnodu et al., 2020; Oron et al., 2020).

O tempo de seguimento dos pacientes apresentou grande variabilidade e apenas dois acompanharam as crianças até a idade adulta (Telfer et al., 2007; Serjeant et al., 2018), o que demonstra a necessidade de estudos longitudinais que avaliem a sobrevida de indivíduos até essa idade, uma vez que estes necessitam ser melhor explorados para maior compreensão da doença, principalmente em localidades de alta prevalência da doença e difícil acesso diagnóstico e de tratamento (Hankins et al., 2018; Wonkam \& Makani, 2019).

Entre a população pesquisada nos estudos, a maior parte apresentava genótipo HbSS, dado que se repetiu na avaliação dos óbitos, onde 68,21\% ocorreram entre indivíduos homozigotos. Houve diferença entre a mediana de sobrevida calculada para o genótipo $\mathrm{SS} / \mathrm{S} \beta^{\circ}$ em relação aos demais.

Estes achados reforçam a certeza de maior prevalência, gravidade e mortalidade associada ao genótipo HbSS (Sabarense, Lima, Silva, \& Viana, 2015; Gardner et al., 2016). O manejo requer diagnóstico e tratamento precoces a fim de prevenir ou minimizar as complicações decorrentes da condição clínica da doença (M. Houwing et al., 2019; Abboud, 2020).

Em relação à sobrevida estratificada por sexo, esta foi discretamente maior entre o masculino (77,5\%), dado também encontrado em outros estudos que evidenciaram uma maior mortalidade, e por consequência uma menor sobrevida, entre indivíduos do sexo feminino (Araujo et al., 2015; Ramos, de Amorim, Pedroso, Nunes, \& Rios, 2015).

A estratificação por níveis de hemoglobina demonstrou resultados diretamente proporcionais à sobrevida, valores de $10 \mathrm{~g} / \mathrm{dL}$ foram associados a uma maior sobrevida ao serem comparados a valores de $6 \mathrm{~g} / \mathrm{dL}$ aos 30 anos. Tal como observado em um estudo em que a cada diminuição de g/dL de Hb o risco de morrer aumentou em 1,2 vezes (Elmariah et al., 2014).

Embora observado que a sobrevida diminua drasticamente após os 30 anos de idade nos pacientes com DF, todos os estudos analisados referiram melhora desta ao serem avaliadas as décadas de acompanhamento. Foi possível observar um incremento na mediana de sobrevida ao se comparar as coortes acompanhadas antes e após o ano de 1981, nas quais para o primeiro período foi de $85 \%$ aos 10 anos de idade, com aumento de $10 \%$ para o período após 1981, com probabilidade de sobrevida de $95 \%$.

O incremento na mediana de sobrevida para DF com o passar dos anos está relacionada com a ampliação das possibilidades diagnósticas e terapêuticas, como a profilaxia com uso de penicilina e imunobiológicos, hemotransfusões, transplante de células tronco hematopoiéticas, terapia com hidroxiureia (HU) (Araujo et al., 2015) e mais recentemente a introdução de novos fármacos (Ballas, 2020). 
Embora tenha ocorrido melhoria na sobrevida dos pacientes com DF ao passar dos anos, a expectativa de vida ajustada pela qualidade de vida chega a ser a metade para indivíduos com DF se comparado com os sem DF (33 versus 67 anos) (Lubeck et al., 2019). Este fato ressalta a necessidade de maior acesso dos pacientes ao atendimento com modelos de tratamento e programas abrangentes, contínuos e personalizados, os quais podem impactar diretamente e positivamente na mortalidade (Sarat et al., 2019; Kanter et al., 2020) e proporcionar maior sobrevida.

A disparidade de acesso aos cuidados especializados, de garantia de seguimento em longo prazo e de tratamento gratuito e de qualidade, pode interferir diretamente no desfecho da doença (L. Lee, Smith-Whitley, Banks, \& Puckrein, 2019; Odame \& Jain, 2020).

Além do diagnóstico precoce obtido por meio dos programas de triagem neonatal, a criação de estratégias de acompanhamento, com mecanismos de busca ativa dos pacientes com consultas agendadas, de modo a minimizar o abandono do seguimento; a garantia de avaliação clínico-laboratorial e tratamento, com acesso aos serviços de saúde e à hidroxiureia de forma gratuita, podem contribuir na melhoria da sobrevida e na diminuição da morbimortalidade (Mbiya et al., 2020; M. E. Houwing et al., 2021).

A gestão de cuidados de saúde com alocação de recursos governamentais e de ações da comunidade podem auxiliar na inserção e monitoramento dessas e de outras intervenções como educação para os pais e pacientes, profilaxia para infecções com antibioticoterapia e imunobiológicos, suplementação com ácido fólico, além de alimentação adequada desde os primeiros meses de vida (Hulihan, Hassell, Raphael, Smith-Whitley, \& Thorpe, 2017; Mburu \& Odame, 2019).

Um estudo realizado na República Democrática do Congo observou que a criação de um centro de referência gratuito para acompanhamento regular dos pacientes com DF com consultas mensais, avaliação clínica e laboratorial, bem como terapias de profilaxia contra infecções, foi responsável pela diminuição do número de complicações clínicas, transfusões e internações hospitalares, fatores que impactam diretamente na morbimortalidade e sobrevida da doença (Mbiya et al., 2020).

A avaliação do risco de viés, por meio da aplicação da escala NOS (Wells et al., 2014) indicou que seis estudos (A. Lee et al., 1995; Quinn et al., 2004; King et al., 2007; Telfer et al., 2007; Quinn et al., 2010; Serjeant et al., 2018) apresentaram pontuação igual ou superior a sete, considerados de alta qualidade. Desta forma as evidências indicam a melhoria da sobrevida quando existe o diagnóstico da DF durante o período neonatal, com acompanhamento clínico subsequente para propiciar tratamento adequado, embora sem a análise do contexto socioeconômico. Mesmo assim, se faz necessário o desenvolvimento de novos estudos longitudinais relativos a DF, para melhor elucidação dos casos.

Entre as principais limitações desta pesquisa esteve a escassez de estudos que abordaram a temática da sobrevida de indivíduos com DF, em especial aos diagnosticados durante o período neonatal. Os estudos encontrados englobam a sobrevida relacionada principalmente aos fatores biológicos da doença e não contemplam os aspectos sociais e econômicos, que podem influenciar diretamente a qualidade de vida desta população. Além disso, não foi possível a realização de metanálise em decorrência da heterogeneidade dos dados com relação ao tempo de seguimento dos participantes. Assim, esta revisão pode contribuir com o aumento das informações acerca do tema e direcionar o cuidado da enfermagem e das autoridades sanitárias para a melhoria da qualidade de vida e por consequência para uma melhor sobrevida e longevidade da população com DF.

\section{Conclusão}

Há necessidade do desenvolvimento de estudos longitudinais que acompanhem os pacientes com DF ao longo de toda vida. Mesmo assim existem evidências de que ocorre melhoria da sobrevida quando os casos de AF são diagnosticados durante o período neonatal. 
Observou-se que a maioria dos casos são de genótipo HbSS, assim como naqueles com desfechos de óbito. Foi encontrada uma sobrevida discretamente maior para o sexo masculino e também associada os valores maiores de hemoglobina sérica, em torno de $10 \mathrm{~g} / \mathrm{dL}$.

Houve uma diminuição da sobrevida após os 30 anos de idade, mas apesar da DF apresentar curso grave e implicar em limitações aos indivíduos, a sobrevida global tem aumentado de forma considerável com o passar dos anos.

Esta condição está intimamente ligada a melhorias de acesso a saúde, com triagem neonatal e tratamentos contínuos com vistas ao aumento da qualidade e sobrevida dos pacientes, os quais muitas vezes estão em condições de vulnerabilidade. A criação de políticas públicas com inserção da comunidade no contexto de suas ações pode auxiliar tanto na manutenção das estratégias que foram pactuadas entre os gestores, quanto na sensibilização de familiares, pacientes e demais membros dessa comunidade, por meio da educação em saúde acerca da DF e suas limitações biológicas, sociais e econômicas.

\section{Referências}

Abboud, M. R. (2020). Standard management of sickle cell disease complications. Hematol Oncol Stem Cell Ther. https://pubmed.ncbi.nlm.nih.gov/32202245/

Araujo, O. M. R., Ivo, M. L., Ferreira Júnior, M. A., Pontes, E. R. J. C., Bispo, I. M. G. P., \& Oliveira, E. C. L. d. (2015). Survival and mortality among users and non-users of hydroxyurea with sickle cell disease. Rev Lat Am Enfermagem, 23, 67-73. http://dx.doi.org/10.1590/0104-1169.3385.2526

Ballas, S. K. (2020). The evolving pharmacotherapeutic landscape for the treatment of Sickle Cell Disease. Mediterr J Hematol Infect Dis, 12(1). https://pubmed.ncbi.nlm.nih.gov/31934320/

Brasil. (2002). Manual de Normas Técnicas e Rotinas Operacionais do Programa Nacional de Triagem Neonatal (pp. 90). Retrieved from https://bvsms.saude.gov.br/bvs/publicacoes/triagem_neonatal.pdf

Brasil. (2005). Portaria no 1.391 de 16 de Agosto de 2005: Institui no âmbito do Sistema Único de Saúde-SUS, as diretrizes para a Política Nacional de Atenção Integral às Pessoas com Doença Falciforme e outras Hemoglobinopatias.Brasília: Ministério da Saúde.

Elmariah, H., Garrett, M. E., De Castro, L. M., Jonassaint, J. C., Ataga, K. I., Eckman, J. R., Ashley-Koch, A. E., \& Telen, M. J. (2014). Factors associated with survival in a contemporary adult sickle cell disease cohort. Am J Hematol, 89(5), 530-535. https://pubmed.ncbi.nlm.nih.gov/24478166/

Fernandes, A. P. P. C., Nélio Januário, J., Blanco Cangussu, C., Lino de Macedo, D., \& Borato Viana, M. (2010). Mortality of children with sickle cell disease: A population study. J Pediatr (Rio J), 86(4). http://dx.doi.org/10.1590/S0021-75572010000400006

Gardner, K., Douiri, A., Drasar, E., Allman, M., Mwirigi, A., Awogbade, M., \& Thein, S. L. (2016). Survival in adults with sickle cell disease in a highincome setting. Blood, 128(10), 1436-1438. https://pubmed.ncbi.nlm.nih.gov/27439910/

Hankins, J. S., Estepp, J. H., Hodges, J. R., Villavicencio, M. A., Robison, L. L., Weiss, M. J., Kang, G., Schreiber, J. E., Porter, J. S., \& Kaste, S. C. (2018). Sickle Cell Clinical Research and Intervention Program (SCCRIP): A lifespan cohort study for sickle cell disease progression from the pediatric stage into adulthood. Pediatr Blood Cancer, 65(9), e27228. https://pubmed.ncbi.nlm.nih.gov/29797644/

Houwing, M., de Pagter, P., van Beers, E., Biemond, B., Rettenbacher, E., Rijneveld, A., Schols, E., Philipsen, J., Tamminga, R., \& van Draat, K. F. (2019). Sickle cell disease: Clinical presentation and management of a global health challenge. Blood Rev, 37, 100580. https://pubmed.ncbi.nlm.nih.gov/31128863/

Houwing, M. E., Buddenbaum, M., Verheul, T. C., de Pagter, A. P., Philipsen, J. N., Hazelzet, J. A., \& Cnossen, M. H. (2021). Improving access to healthcare for paediatric sickle cell disease patients: a qualitative study on healthcare professionals' views. BMC Health Serv Res, 21(1), 1-13. https://pubmed.ncbi.nlm.nih.gov/33712013/

Hulihan, M., Hassell, K. L., Raphael, J. L., Smith-Whitley, K., \& Thorpe, P. (2017). CDC Grand Rounds: Improving the Lives of Persons with Sickle Cell Disease. MMWR. Morbidity and mortality weekly report, 66(46), 1269-1271. https://pubmed.ncbi.nlm.nih.gov/29166365/ doi:10.15585/mmwr.mm6646a2

Institute for Health Metrics and Evaluation. (2018). Both Sexes, All ages, 1990-2016, YLLs per 100.000. http://www.healthdata.org/

Kanter, J., Smith, W. R., Desai, P. C., Treadwell, M., Andemariam, B., Little, J., Nugent, D., Claster, S., Manwani, D. G., \& Baker, J. (2020). Building access to care in adult sickle cell disease: defining models of care, essential components, and economic aspects. Blood Adv, 4(16), 3804-3813. https://pubmed.ncbi.nlm.nih.gov/32785684/

Kikuchi, B. A., Ivo, M. L., Barbieri, A. R., Camargo Filho, R., Amargo Filho, R., \& Nascimento, V. (2018). Evaluation of the implantation of the national neonatal screening program regarding coverage index, disease prevalence and sickle cell trait in Mato Grosso do Sul-Brazil: 2001-2015. IJDR, 8(3), 1927919283. https://www.journalijdr.com/evaluation-implantation-national-neonatal-screening-program-regarding-coverage-index-disease

King, L., Fraser, R., Forbes, M., Grindley, M., Ali, S., \& Reid, M. (2007). Newborn sickle cell disease screening: the Jamaican experience (1995-2006). J Med Screen, 14(3), 117-122. https://pubmed.ncbi.nlm.nih.gov/17925083/

Kloda, L. A., \& Bartlett, J. C. (2013). Formulating answerable questions: question negotiation in evidence-based practice. J Can Health Libr Assoc, 34(2), 5560. https://journals.library.ualberta.ca/jchla/index.php/jchla/article/view/22636 
Lê, P.-Q., Ferster, A., Dedeken, L., Vermylen, C., Vanderfaeillie, A., Rozen, L., Heijmans, C., Huybrechts, S., Devalck, C., \& Cotton, F. (2018). Neonatal screening improves sickle cell disease clinical outcome in Belgium. J Med Screen, 25(2), 57-63. DOI: 10.1177/0969141317701166

Lee, A., Thomas, P., Cupidore, L., Serjeant, B., \& Serjeant, G. (1995). Improved survival in homozygous sickle cell disease: lessons from a cohort study. Bmj, 311(7020), 1600-1602. https://pubmed.ncbi.nlm.nih.gov/8555802/

Lee, L., Smith-Whitley, K., Banks, S., \& Puckrein, G. (2019). Reducing Health Care Disparities in Sickle Cell Disease: A Review. Public Health Reports, 134(6), 599-607. https://pubmed.ncbi.nlm.nih.gov/31600481/ doi:10.1177/0033354919881438

Lubeck, D., Agodoa, I., Bhakta, N., Danese, M., Pappu, K., Howard, R., Gleeson, M., Halperin, M., \& Lanzkron, S. (2019). Estimated Life Expectancy and Income of Patients With Sickle Cell Disease Compared With Those Without Sickle Cell Disease. JAMA Netw Open, $2(11)$, e1915374-e1915374. https://pubmed.ncbi.nlm.nih.gov/31730182/

Mallmann, M. B., Tomasi, Y. T., \& Boing, A. F. (2020). Realização dos testes de triagem neonatal no Brasil: prevalências e desigualdades regionais e socioeconômicas. J. Pediatr, 96(4), 487-494. http://dx.doi.org/10.1016/j.jped.2019.02.008

Mbiya, B. M., Kalombo, D. K., Mukendi, Y. N., Mpoyi, J. K., Biboyi, P. M., Daubie, V., Disashi, G. T., \& Gulbis, B. (2020). Improvement of Sickle Cell Disease Morbimortality in Children: Experience in a Remote Area of an African Country. Research Square. doi: 10.20944 / preprints202005.0305.v2

Mburu, J., \& Odame, I. (2019). Sickle cell disease: Reducing the global disease burden. Int J Lab Hematol, 41(S1), 82-88. https://doi.org/10.1111/ijlh.13023

McGann, P. T. (2016). Time to invest in sickle cell anemia as a global health priority. Pediatrics, 137(6). https://pubmed.ncbi.nlm.nih.gov/27244863/

Moher, D., Shamseer, L., Clarke, M., Ghersi, D., Liberati, A., Petticrew, M., Shekelle, P., \& Stewart, L. A. (2015). Preferred reporting items for systematic review and meta-analysis protocols (PRISMA-P) 2015 statement. Syst Rev, 4(1), 1. https://pubmed.ncbi.nlm.nih.gov/25554246/

Nnodu, O. E., Sopekan, A., Nnebe-Agumadu, U., Ohiaeri, C., Adeniran, A., Shedul, G., Isa, H. A., Owolabi, O., Chianumba, R. I., \& Tanko, Y. (2020). Implementing newborn screening for sickle cell disease as part of immunisation programmes in Nigeria: a feasibility study. Lancet Haematol, 7(7), e534-e540. https://pubmed.ncbi.nlm.nih.gov/32589979/

Odame, I., \& Jain, D. (2020). Sickle cell disease: Progress made \& challenges ahead. Indian J Med Res, 151(6), 505. https://pubmed.ncbi.nlm.nih.gov/32719221/

Oron, A. P., Chao, D. L., Ezeanolue, E. E., Ezenwa, L. N., Piel, F. B., Ojogun, O. T., Uyoga, S., Williams, T. N., \& Nnodu, O. E. (2020). Caring for Africa's sickle cell children: will we rise to the challenge? BMC Med, 18, 1-8. https://pubmed.ncbi.nlm.nih.gov/32340612/

Ouzzani, M., Hammady, H., Fedorowicz, Z., \& Elmagarmid, A. (2016). Rayyan-a web and mobile app for systematic reviews. Syst Rev, 5(1), 210. https://pubmed.ncbi.nlm.nih.gov/27919275/

Piel, F. B., Hay, S. I., Gupta, S., Weatherall, D. J., \& Williams, T. N. (2013). Global burden of sickle cell anaemia in children under five, 2010-2050: modelling based on demographics, excess mortality, and interventions. PLoS Med, 10(7), e1001484. https://pubmed.ncbi.nlm.nih.gov/23874164/

Piel, F. B., Steinberg, M. H., \& Rees, D. C. (2017). Sickle cell disease. $N \quad$ Engl J Med, $376(16)$, 1561-1573. https://www.nejm.org/doi/full/10.1056/NEJMra1510865?url_ver=Z39.88-2003\&rfr_id=ori:rid:crossref.org\&rfr_dat=cr_pub\%3dpubmed

Quinn, C. T., Rogers, Z. R., \& Buchanan, G. R. (2004). Survival of children with sickle cell disease. Blood, 103(11), 4023-4027. https://doi.org/10.1182/blood-2003-11-3758

Quinn, C. T., Rogers, Z. R., McCavit, T. L., \& Buchanan, G. R. (2010). Improved survival of children and adolescents with sickle cell disease. Blood, 115(17), 3447-3452. https://doi.org/10.1182/blood-2009-07-233700

Ramos, J. T., de Amorim, F. S., Pedroso, F. K. F., Nunes, A. C. C., \& Rios, M. A. (2015). Mortalidade por doença falciforme em estado do nordeste brasileiro. R. Enferm. Cent. O. Min. http://www.seer.ufsj.edu.br/index.php/recom/article/view/859

Runkel, B., Klüppelholz, B., Rummer, A., Sieben, W., Lampert, U., Bollig, C., Markes, M., Paschen, U., \& Angelescu, K. (2020). Screening for sickle cell disease in newborns: a systematic review. Syst Rev, 9(1), 1-9. doi: 10.1186/s13643-020-01504-5

Sabarense, A. P., Lima, G. O., Silva, L. M., \& Viana, M. B. (2015). Characterization of mortality in children with sickle cell disease diagnosed through the Newborn Screening Program. J Pediatr (Rio J), 91(3), 242-247. https://pubmed.ncbi.nlm.nih.gov/25449790/

Sarat, C. N. F., Ferraz, M. B., Júnior, M. A. F., Souza, A. S. d., Cardoso, A. I. d. Q., \& Ivo, M. L. (2019). Prevalence of sickle cell disease in adults with delayed diagnosis. Acta Paul Enferm, 32(2), 202-209. DOI: 10.1590/1982-0194201900028

Serjeant, G. R., Chin, N., Asnani, M. R., Serjeant, B. E., Mason, K. P., Hambleton, I. R., \& Knight-Madden, J. M. (2018). Causes of death and early life determinants of survival in homozygous sickle cell disease: The Jamaican cohort study from birth. PloS one, 13(3), e0192710. https://pubmed.ncbi.nlm.nih.gov/29494636/

Stroup, D. F., Berlin, J. A., Morton, S. C., Olkin, I., Williamson, G. D., Rennie, D., Moher, D., Becker, B. J., Sipe, T. A., \& Thacker, S. B. (2000). Metaanalysis of observational studies in epidemiology: a proposal for reporting. Jama, 283(15), 2008-2012. https://pubmed.ncbi.nlm.nih.gov/10789670/

Sundd, P., Gladwin, M. T., \& Novelli, E. M. (2019). Pathophysiology of sickle cell disease. Annu Rev Pathol, 14, 263-292. 10.1146/annurev-pathmechdis012418-012838

Telfer, P., Coen, P., Chakravorty, S., Wilkey, O., Evans, J., Newell, H., Smalling, B., Amos, R., Stephens, A., \& Rogers, D. (2007). Clinical outcomes in children with sickle cell disease living in England: a neonatal cohort in East London. haematologica, 92(7), 905-912. http://www.haematologica.org/content/92/7/905.long 
Research, Society and Development, v. 10, n. 11, e95101119329, 2021

(CC BY 4.0) | ISSN 2525-3409 | DOI: http://dx.doi.org/10.33448/rsd-v10i11.19329

Ware, R. E., de Montalembert, M., Tshilolo, L., \& Abboud, M. R. (2017). Sickle cell disease. Lancet, 390(10091), 311-323. https://www.thelancet.com/journals/lancet/article/PIIS0140-6736(17)30193-9/fulltext

Wells, G., Shea, B., O’Connell, D., Peterson, J., Welch, V., Losos, M., \& Tugwell, P. (2014). Newcastle-Ottawa quality assessment scale cohort studies. 15 http://www.ohri.ca/programs/clinical_epidemiology/oxford.asp

Wonkam, A., \& Makani, J. (2019). Sickle cell disease in Africa: an urgent need for longitudinal cohort studies. Lancet Glob Health, 7(10), e1310-e1311. https://pubmed.ncbi.nlm.nih.gov/31451442/ 